\title{
Improved Voltage Harmonics Compensation Strategy for a Stand-Alone DFIG Operating under Unbalanced and Nonlinear Load Conditions
}

\author{
Kaddour Abdoune ${ }^{1 *}$, Fateh Abdoune', Djamal Aouzellag ${ }^{1}$, Kaci Ghedamsi ${ }^{1}$ \\ ${ }^{1}$ Laboratoire de Maitrise des Energies Renouvelables, Faculté de Technologie, Université de Bejaia, 06000 Bejaia, Algeria \\ * Corresponding author, e-mail: kaddour_abdoune@yahoo.fr
}

Received: 27 September 2019, Accepted: 11 November 2019, Published online: 13 January 2020

\begin{abstract}
The study presents an improved control and operation for a stand-alone Doubly-Fed Induction Generator (DFIG) energy system under unbalanced and nonlinear load conditions. This type of loads results in unbalance and distorted stator voltage and current at the Point of Common Coupling (PCC), with subsequent power quality degradation and electromagnetic torque pulsations of DFIG. A new and simple control strategy based Proportional-Resonant Controllers (PRCs) in the stationary reference frame is applied in the Load-Side Converter (LSC) for fully compensate the unbalance and harmonic distortion in the PCC and avoid the torque pulsations of DFIG. As a result, symmetrical and sinusoidal AC voltage is applied to the PCC, which indicates that the Rotor-Side Converter (RSC) can be still controlled with the traditional vector control strategy, to adjust the generator torque and to balance active power. This strategy is conceptually simple, it does not require for rotating coordinate transformation and the sequence decomposition process, in addition no current sensors need to be installed at load or stator side, so that the number of current sensors employed is reduced. Simulation results using MatLab/Simulink are given and show the effectiveness and validity of the proposed control scheme to eliminate the harmonics in the PCC and torque pulsations of DFIG.
\end{abstract}

Keywords

stand-alone system, Doubly-Fed Induction Generator (DFIG), unbalanced and nonlinear loads, harmonics compensation, torque ripples reduction, Proportional-Resonant Controllers (PRC)

\section{Introduction}

Nowadays, doubly-fed induction generators are receiving renewed attention as sources of constant frequency generators from variable speed movers. The use of DFIG in energy conversion systems gives the advantage that is the only scheme in which the output power generated is greater than the nominal power of the stator of the employed machine $[1,2]$. This advantage results in increasing the efficiency of generation. Also, the wide range of speed within which active power could be generated makes the DFIG more efficient and suitable for use in conjunction with the variable speed generation systems, such as diesel engines, wind turbines, and water turbines.

In the recent decade, the control and operation of DFIG systems under grid-connected energy conversion applications has been the focus of much of research, including the decoupled active and reactive power control [1,3], modeling and control $[2,4]$, unbalanced grid network $[5,6]$, and distorted grid voltage $[6,7]$. However, the technical publications associated with stand-alone DFIG applications are less, while the power supplies system for ships, remote villages, military equipment, etc., requires standalone generation systems to meet the electricity demand. The most important characteristic of a stand-alone DFIG is that the system itself must be able to generate a constant voltage and frequency in the stator side irrespective of the rotational speed and the loading conditions [8-10]. But when unbalanced or non-linear loads are connected to the PCC, stator voltage and current can be unbalanced or distorted, which would introduce torque pulsations in the generators. In such conditions, the efficiency of the generators will largely reduce, the power quality of the loads is degraded and the performance of other loads will be affected. Therefore, it is desirable to eliminate the negative phase sequence and harmonic components of the stator 
voltage and current in order to overcome such deficiencies and ensure good power quality.

Several works have proposed different control techniques which aim to compensate unbalanced and harmonic components in the DFIG system in case of imbalanced and nonlinear loads are supplied. In [11], a traditional ProportionalIntegral (PI) controllers designed based on the synchronous reference frame is used to compensate unbalance and load harmonics currents. However, using PI has major disadvantages such as steady-state errors and the small gains at the harmonic frequencies. Consequently, the unbalanced and harmonic components are extracted using band-pass filters (BPFs) and then transformed into their corresponding reference frames $[12,13]$. Unfortunately, this method is complex and requires a large number of calculations, because the elimination of negative sequence and each given harmonic component requires a BPF. In addition, the extraction process of harmonic components could lead to instability in the control current loop. So, the effectiveness of this method is limited.

Alternatively, improved control strategies based PI regulators with resonant loops applied in stationary or synchronous frame are proposed for rotor current control [5, 6 , 10, 14-18]. The proposed control schemes achieve effectiveness in harmonics components reducing and negative sequence eliminating of the stator voltage at the PCC. However, such methods couldn't simultaneously mitigate the power/torque pulsations and balance the stator/rotor currents. To deal with this problem, a compensation method based PI with resonant controllers is developed to compensate unbalanced and distorted stator currents through the LSC, and so to eliminate unbalanced and harmonic voltages at the PCC through the RSC [19-21]. Such control schemes remain complex for implementation because harmonic elimination is applied in two sides (RSC and LSC) of the machine and it requires notch filters for the negative and harmonic components decomposition. In addition, current sensors need to be installed at load or stator side for the purpose of stator harmonics currents compensation.

As opposed to the control methods described above, in this work, a new and simple control strategy for standalone DFIG under unbalanced and nonlinear load conditions with reduced current sensors is proposed. In this strategy, the LSC is controlled to regulate reactive power required by the load, for sinusoidal reference tracking control and for fully reject the low-order harmonic components and the negative sequence of the stator voltage.
As a result, balanced and linear AC voltage is applied to the stator terminal at the PCC, which indicates that the RSC can be still controlled with the conventional vector control strategy, to keep active power balance and constant DC-link voltage. A cascaded current-voltage control loop based on the stationary reference coordinate is proposed for the control of LSC, in which the closed loop voltage regulation is developed by employing proportional and multiple resonant regulators tuned at a low-order harmonic frequency in the stationary reference frame, without any coordinate transformation and notch filter for the negative sequence and harmonic voltage components decomposition. So, this strategy is simple and computational effort is decreased. Indirect stator flux oriented control is applied for the RSC, which requires not additional current sensors to be installed at load or stator side.

\section{General system description and modeling}

Fig. 1 shows a typical structure of stand-alone DFIG system, where loads, including nonlinear and unbalanced loads, are connected to the stator terminals. With the connection of such loads, the voltage at the PCC and the stator current of the DFIG would be unbalanced and distorted. The stator is directly connected to the terminal loads which make the DFIG more sensitive to the harmonics. The rotor is interfaced through a back-to-back Pulse Width Modulation (PWM) Voltage Source Converter (VSC) composed of the RSC and the LSC with a common DC-link capacitor. In addition, a filtering capacitor is installed in the stator terminals at the PCC for allows carrying out a three-phase voltage source, reducing stator output voltage ripples, providing a part of excitation current for the DFIG and filtering high-frequency components produced by PWM converters. The rotor speed is governed by RSC in order to balance the power demanded by the loads with the power taken from the mechanical system, while the stator voltage regulation and compensation is achieved by LSC. The modeling of the focal components of the studied power system and the detail of the control philosophy are explained below. The positive polarity convention of important system variables is shown in Fig. 1.

\subsection{DFIG model}

Considering symmetrical and isentropic magnetic conditions, the dynamic behavior of the three-phase wound rotor induction machine in the $d$ - $q$ synchronous rotating reference frame is described by: 


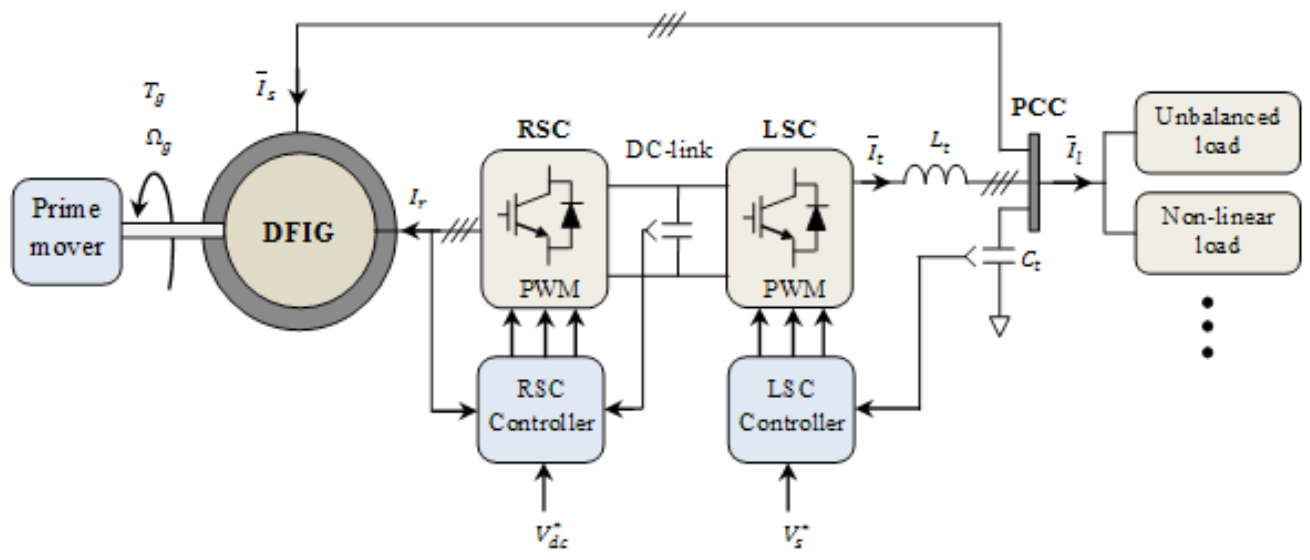

Fig. 1 Global configuration of stand-alone DFIG under study

$$
\begin{aligned}
& \bar{V}_{s}=R_{s} \cdot \bar{I}_{s}+\frac{d}{d t} \bar{\phi}_{s}+j \cdot \omega_{s} \cdot \bar{\phi}_{s} \\
& \bar{V}_{r}=R_{r} \cdot \bar{I}_{r}+\frac{d}{d t} \bar{\phi}_{r}+j \cdot \omega_{r} \cdot \bar{\phi}_{r} .
\end{aligned}
$$

The stator and rotor flux linkages could be determined using:

$$
\begin{aligned}
& \bar{\phi}_{s}=L_{s} \cdot \bar{I}_{s}+L_{m} \cdot \bar{I}_{r} \\
& \bar{\phi}_{r}=L_{r} \cdot \bar{I}_{r}+L_{m} \cdot \bar{I}_{s},
\end{aligned}
$$

where superscript $\left(^{-}\right.$) denotes the vector form of flux linkage, voltage and current. The parameters $L_{s}$ and $L_{r}$ represent respectively the stator and rotor winding cyclic inductances, $L_{m}$ is the mutual cyclic inductance between stator and rotor windings which is kept constant in linear modeling.

The electromagnetic torque can be written as a function stator and rotor current vectors:

$T_{g}=p \cdot L_{m} \cdot \operatorname{Im}\left(\bar{I}_{s} \cdot \bar{I}_{r}^{*}\right)$,

where superscript $\left(^{*}\right)$ denotes the complex conjugate operator, $(\mathrm{Im})$ represents the imaginary component of the result and $p$ is machine pole pair number.

\subsection{LC filter model}

As shown in Fig. 2, the LSC ensures the power transfer from the DC bus to the AC load through an LC filter. This filter plays a role in eliminating harmonic components of the output voltage caused by high-frequency switching actions and allows carrying out a three-phase source by controlling voltages on capacitors terminals.

Using the notation and current flow of the Fig. 2, the Eqs. (6) and (7) describing the AC filter dynamics for each phase can be written as

$i_{t}=\frac{1}{L_{t}} \cdot \int\left(v_{s}-v_{t}\right) d t$

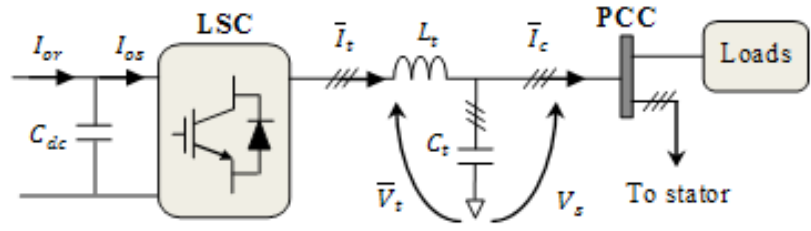

Fig. 2 Equivalent circuit of the LSC and DC side circuitry

$v_{s}=\frac{1}{C_{t}} \cdot \int\left(i_{t}-i_{c}\right) d t$,

where $v_{t}$ is the output phase voltage of the converter, $v_{s}$ is the load bus phase voltage, $i_{t}$ is the current of the inductance, $i_{c}$ is the converter load-side current.

\subsection{VSCs and DC side circuitry}

The functional model of the simulated three-phase converter is used [1]. Using the notation and current flow definitions of the Fig. 3 (a), the voltages of AC and DC side of each VSC are related as

$$
\begin{aligned}
& v_{r a}=\frac{1}{3} \cdot\left(2 \cdot \lambda_{a}-\lambda_{b}-\lambda_{c}\right) \cdot V_{d c} \\
& v_{r b}=\frac{1}{3} \cdot\left(2 \cdot \lambda_{b}-\lambda_{c}-\lambda_{a}\right) \cdot V_{d c} \\
& v_{r c}=\frac{1}{3} \cdot\left(2 \cdot \lambda_{c}-\lambda_{a}-\lambda_{b}\right) \cdot V_{d c} .
\end{aligned}
$$

The total DC current can be computed by doing a power balance on each converter

$$
I_{o r}=\lambda_{a} \cdot i_{r a}+\lambda_{b} \cdot i_{r b}+\lambda_{c} \cdot i_{r c}
$$

where $\lambda_{a}, \lambda_{b}$ and $\lambda_{c}$ are the corresponding switching functions of the converter legs. Any of the three switching functions takes the value of "1" if upper switch in the given converter leg is on and lower switch is off, and value "0" if lower switch in the same converter leg is on, while upper switch is off. The VSC is switched by Space Vector Pulse-Width 

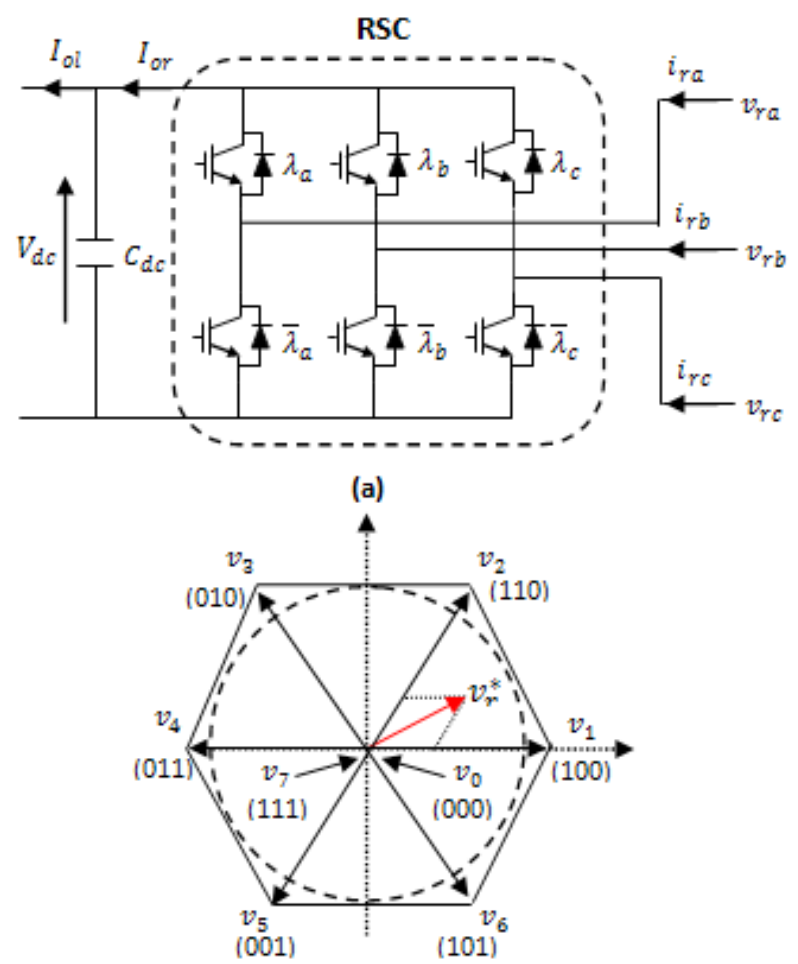

(b)

Fig. 3 Power circuit of the VSC and discrete representation of the VSC output voltage vector

Modulation (SVPWM) technique, it can assume eight (8) different switching states $\left(\lambda_{a}, \lambda_{b}, \lambda_{c}\right)$ corresponding to seven (7) discrete voltage vectors output, as shown in Fig. 3 (b).

The DC-link circuitry is a coupling and a storage element for the energy flowing between the LSC and the RSC. It is represented by a capacitor which serves as a source of continuous energy between two converters. The dynamics of the voltage across the capacitor is given in Eq. (10):

$V_{d c}=\frac{1}{C_{d c}} \cdot \int\left(I_{o r}-I_{o s}\right) d t$,

where $C_{d c}$ is the circuit capacitance, $I_{o s}$ and $I_{o r}$ are respectively the current through the DC side of the stator and the rotor converter.

\section{DFIG harmonics problem analysis}

As the loads are mostly single-phase and nonlinear in the distribution systems, the current in different phases will not be the same and will be distorted. These loads connected to the PCC leads to the presence of negative sequence and harmonic components in the stator current, causes unbalanced and distorted stator voltages at the PCC and induce electromagnetic torque pulsations [20, 22]. If such unbalance and distorted are not taken into account, the voltage unbalance factor (i.e., ratio of negative to the positive sequence of the fundamental component) and Total Harmonic Distortion (THD) may not be within the permissible limit. Therefore, the power quality of the DFIG seriously will be reduced.

An unbalanced three-phase power system can be represented by the superposition of three balanced systems, known as the positive sequence circuit, negative sequence circuit, and zero sequence circuit. Since the zero-sequence stator currents will not induce a torque under the unbalanced stator conditions [22], the stator voltage comprises a positive sequence and negative sequence, so are the stator and rotor current quantities. The stator and rotor currents have two components: positive-sequence components $\left(\bar{I}_{s+}, \bar{I}_{r+}\right)$ and negative-sequence components $\left(\bar{I}_{s-}, \bar{I}_{r-}\right)$ tuned at double synchronous frequency. Stator and rotor vector currents can be written as

$$
\begin{aligned}
& \bar{I}_{s}=\bar{I}_{s+}+\bar{I}_{s-} e^{-j 2 \omega_{s} t} \\
& \bar{I}_{r}=\bar{I}_{r+}+\bar{I}_{r-} e^{-j 2 \omega_{s} t} .
\end{aligned}
$$

Substituting Eq. (11) and Eq. (12) into Eq. (5) yields the electromagnetic torque expression as

$T_{g}=p \cdot L_{m} \cdot \operatorname{Im}\left(\left(\bar{I}_{s+}+\bar{I}_{s-} e^{-j 2 \omega_{s} t}\right)\left(\bar{I}_{r+}^{*}+\bar{I}_{r-}^{*} e^{j 2 \omega_{s} t}\right)\right)$.

Nonlinear loads such as three phase full bridge diode rectifiers always generate quasi-sine AC current. When such loads are connected to the PCC, the stator current and stator voltage would be distorted due to the nonlinear load current including odd harmonics with order $6 n \pm 1$ multiples of synchronous frequency. The harmonic components would also be presented in the rotor current.

$\bar{I}_{s}=\bar{I}_{s}^{1}+\sum_{n=1}^{\infty} \bar{I}_{s}^{6 n \pm 1} e^{ \pm j 6 n \omega_{s} t}$

$\bar{I}_{r}=\bar{I}_{r}^{1}+\sum_{n=1}^{\infty} \bar{I}_{r}^{6 n \pm 1} e^{ \pm j 6 n \omega_{s} t}$

The reaction between the harmonic component in the rotor current and the harmonic component in the stator current will cause a considerable pulsating torque. According to Eq. (14) and Eq. (15), the electromagnetic torque of the DFIG is given by Eq. (16).

$$
\begin{aligned}
T_{g} & =p \cdot L_{m} \\
& \cdot \operatorname{Im}\left(\left(\bar{I}_{s}^{1}+\sum_{n=1}^{\infty} \bar{I}_{s}^{6 n \pm 1} e^{ \pm j 6 n \omega_{s} t}\right)\left(\bar{I}_{r}^{* 1}+\sum_{n=1}^{\infty} \bar{I}_{r}^{* 6 n \pm 1} e^{\mp j 6 n \omega_{s} t}\right)\right)
\end{aligned}
$$

It is worth noting from Eq. (13) and Eq. (16) that negatives sequences and harmonics components of stator and rotor currents affect the torque, which indicates torque pulsations 
with frequency $2 \omega_{s}$ and $6 n \omega_{s}$ would appear in the generator. This may increase the stress on mechanical components and heat in the generator [23]. In order to avoid these negative impacts, stator and rotor currents harmonics need to be eliminated. Moreover, stator voltage harmonics and imbalance need to be compensated for the purpose of improving the power quality of loads connected at the PCC.

\section{Proposed control scheme}

The proposed control scheme can be divided into machine side control and load side control. Here, the problem of torque pulsations in the machine is solved by regulating sinusoidal and balanced currents in the stator and rotor windings. With the aim to avoid currents distortion and imbalance in the DFIG stator, the negative sequence and ideally any distortion in the three phase loads should be supplied by the LSC. Additionally, the LSC should handle a fraction of the stator positive-sequence current, i.e. active power to achieve regulation of the DC-link voltage and if necessary to provide reactive power required by the load. In such case, the considering of the LSC rating becomes a necessity to ensure its smooth operation with the proposed control.

To achieve disturbance suppression and sinusoidal reference tracking control, a control strategy including PRCs is proposed to be applied in LSC controller for the purpose of PCC voltage regulation. Consequently, the DFIG stator voltage and current at the PCC can be kept symmetrical and sinusoidal by the proposed LSC control. Thus, the RSC can be controlled with the traditional vector control in order to keep constant the DC bus voltage under rotor speed and load disturbances.

\subsection{PCC voltage regulation by $\mathrm{LSC}$}

The control aim of the LSC is to regulate the PCC so as to keep constant voltage and constant frequency in the generation operation, even if unbalanced, nonlinear and heavy load disturbance takes place. With the aim to avoid voltage distortion and imbalance at the PCC, the LSC is controlled to produce a proper output voltage that compensates unbalanced and nonlinear voltage drop. As a result, a balanced and sinusoidal voltage waveform can be produced at the PCC despite the effect of such severe load conditions.

A control strategy including proportional resonant controllers (PRCs) is proposed for the closed-loop PCC voltage regulation. This type of regulators is well adapted to the control of sinusoidal reference signals. They can be effectively combined together to achieve a multiple resonant controller, which allows performing selective harmonic control. The transfer function of a PRC with $m$-frequency elements is expressed as

$C(s)=K_{p}+\sum_{i=1}^{m}\left(\frac{A_{i} s}{s^{2}+2 \omega_{c i}+\omega_{i}^{2}}\right)$,

where $K_{p}$ and $A_{i}$ are the proportional and resonant parameters of the controller, respectively, which can be determined based on the Naslin polynomial [24]. The parameters $\omega_{i}$ and $\omega_{c i}$ are the angular frequencies of regulated terms and cut-off angular frequency respectively.

The selection of resonant frequency for each resonant controller is dependent on which kind of load to be used. In this paper, the fundamental and two harmonics components $\left(5^{\text {th }}\right.$ and $\left.7^{\text {th }}\right)$ are considered as the main voltage harmonic orders. Accordingly, the controller contains three resonant regulators tuned at selected frequencies, that is, the fundamental $\left(\omega_{0}\right)$, the fifth $\left(5 \omega_{0}\right)$ and the seventh $\left(7 \omega_{0}\right)$ multiples of fundamental frequency. The adjustment of the voltage regulator was done by means of zeros and gain manipulation in the root locus using Matlab's SISO design tool. The Bode plot of the open-loop transfer function associated to the PRC is shown in Fig. 4.

The proposed LSC voltage control is shown in Fig. 5. It contains two cascaded loops: an inner current loop to regulate the current in filter inductor and an outer voltage loop to regulate the voltage on the capacitor terminals. The output signal of the voltage closed-loop control with PRCs is directly used as the reference of the current closed control loop. As illustrated in Fig. 5, the control scheme is implemented in the stationary reference frame, the measured three-phase voltages and currents are directly used without any coordinate transformation and
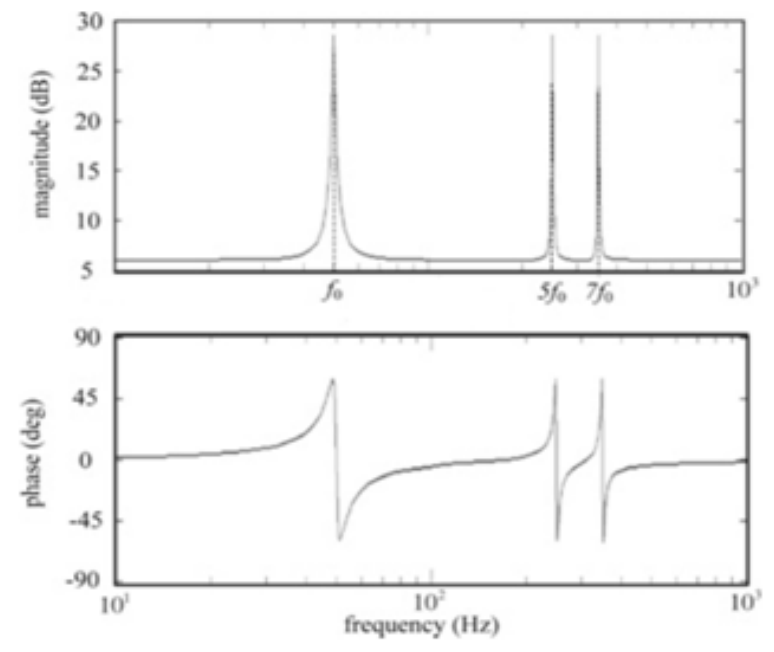

Fig. 4 Bode plot of PRC with three resonant frequencies 


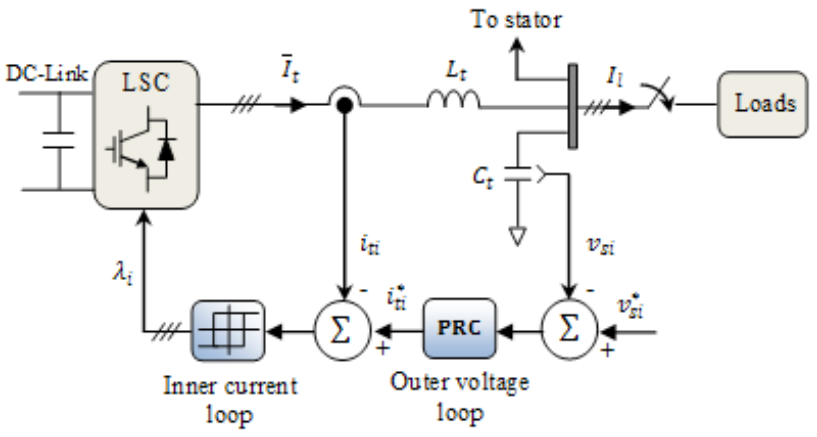

Fig. 5 Proposed LSC control scheme

notch filter for the negative sequence and harmonic voltage component decomposition. The reference output voltages can be described as

$\bar{V}_{s}^{*}=V_{s} \cdot e^{j \omega_{s} t}$,

where $V_{s}$ and $\omega_{s}$ are respectively the phase magnitude and angular frequency of the voltage reference.

Owing to the influence of the negative sequence and harmonic voltages, the generated current references by an outer voltage control loop will contain the fundamental and harmonic components. The current controller can be designed to regulate the current precisely to meet the desired reference values without decomposing the inductor current. Hysteresis controllers are employed for controlling each phase of the inductor current. Each controller determines the switching state of the corresponding inverter leg such that the inductor currents are forced to remain within the hysteresis band. As is known, these controllers promise controllable reference-tracking error and fast response. The only drawback of this approach remains the inherently variable switching frequency [25].

\subsection{RSC analysis and control}

As stated above, the negative-sequence and distorted harmonic stator voltage of DFIG can be suppressed with the proposed control of the LSC, which means that a balanced and undistorted $\mathrm{AC}$ voltage is applied to the stator terminal of the DFIG. Thus, for the RSC, under unbalanced and nonlinear load conditions, the traditional vector control strategy can be used.

The main task of the RSC control is to adjust the DFIG electrical torque according to the shaft speed in order to balance the power demanded by the loads with the power taken from the mechanical system. Vector control is achieved by regulating the rotor currents in a synchronous rotating frame oriented with the stator flux position, following the scheme shown in Fig. 6.

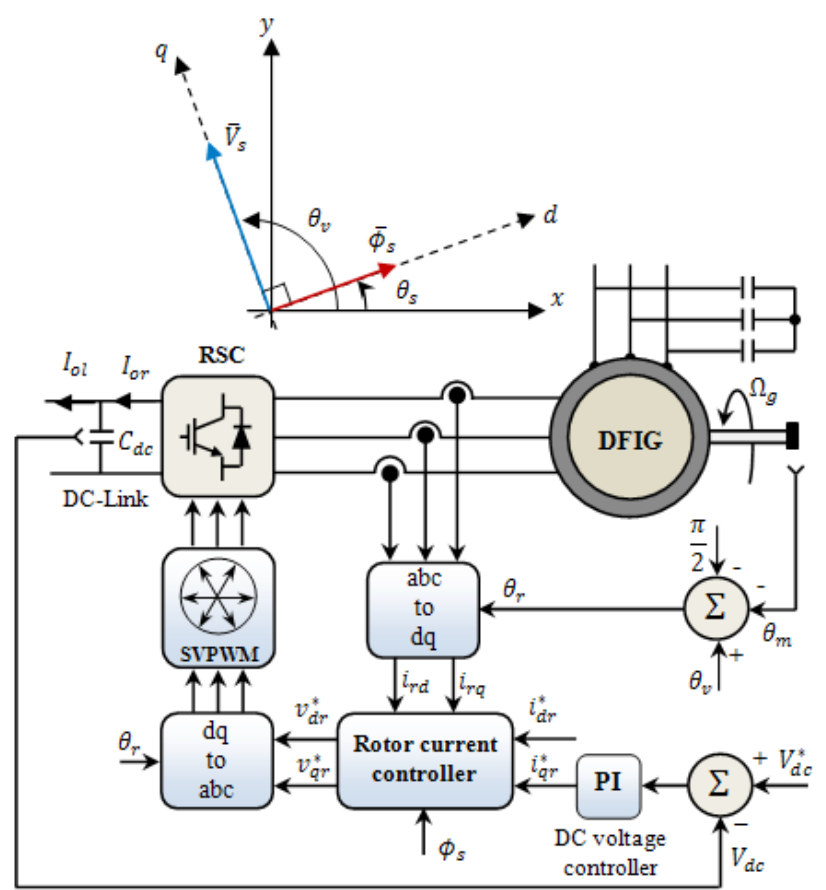

Fig. 6 Proposed vector control schematic of DFIG

According to Eq. (3) and Eq. (4), the rotor flux linkage could be expressed as

$\bar{\phi}_{r}=\frac{L_{m}}{L_{s}} \cdot \bar{\phi}_{s}+\sigma \cdot L_{r} \cdot \bar{I}_{r}$,

where $\sigma=1-\frac{L_{m}^{2}}{L_{s} \cdot L_{r}} \quad$ is the leakage coefficient of the machine.

Substituting Eq. (19) into Eq. (2) yields the rotor voltage in the $d-q$ reference frame as

$\bar{V}_{r}=\left(R_{r}+\sigma \cdot L_{r}\left(\frac{d}{d t}+j \omega_{r}\right)\right) \cdot \bar{I}_{r}+\frac{L_{m}}{L_{s}} \cdot\left(\frac{d}{d t}+j \omega_{r}\right) \cdot \bar{\phi}_{s}$.

Assuming the stator voltage and flux to be constant, the dynamic equations governing the rotor currents in the $d-q$ stator flux coordinates are obtained by decomposition of Eq. (20) into orthogonal components

$v_{r d}=R_{r} \cdot i_{r d}+\sigma \cdot L_{r} \cdot \frac{d i_{r d}}{d t}-\omega_{r} \cdot \sigma \cdot L_{r} \cdot i_{r q}$

$v_{r q}=R_{r} \cdot i_{r q}+\sigma \cdot L_{r} \cdot \frac{d i_{r q}}{d t}+\omega_{r} \cdot \frac{L_{m}}{L_{s}} \cdot \phi_{s d}+\omega_{r} \cdot \sigma \cdot L_{r} \cdot i_{r d} \cdot$

Equations (21) and (22) are the basic for designing the current loop controllers. The $d$-axis and $q$-axis rotor currents are regulated using the PI controllers. Decoupling terms are added to the controller outputs to provide linear transfer functions in order to simplify the controller design and ensure good tracking of these currents. 
According to the chosen field orientation, the $q$-axis rotor current is selected as the variable to be changed to regulate the DC-link voltage, by which enables machine torque production. As shown in Fig. 6, the current reference for $q$-axis is created by means of closed loop DC-voltage control using a PI controller designed according to the model defined by Eq. (10). The inner current control loop can be approximated as a unity gain because the bandwidth is significantly higher than that of the DC link voltage controller loop. When the LSC is injecting an unbalanced or distorted three-phase power to the load, the DC-side current oscillates at two times or at six times the line frequency. If the oscillation is not removed, the bandwidth of the loop must be limited to provide sufficient attenuation of the oscillatory component to avoid distorting the current reference.

The $d$-axis rotor current may be used to supply the magnetizing current to the machine. In the proposed standalone system, the required reactive power for a DFIG to setting up magnetic flux is supplied entirely from the rotor (i.e. the stator reactive power is set to zero), and the reactive power drawn by the load will naturally supplied by the LSC under the action of the stator voltage loop. With this control structure, the magnetization requirements on the rotor side are reduced and the margin of torque creating the current to generate active stator power is improved. Consequently, the losses in the RSC and the windings of the machine decrease and the maximal active power generation can be obtained. The $d$-axis rotor current reference is derived as

$$
i_{d r}^{*}=\frac{V_{s}}{\omega_{s} \cdot L_{m}} \text {. }
$$

The stator flux position angle $\theta_{s}$ needed for the orientation of the frame is obtained from the stator voltage reference. Neglecting the stator resistance, the orientation angle can be calculated in steady state as

$\theta_{s}=\theta_{v}-\frac{\pi}{2}=\int \omega_{s}^{*} d t-\frac{\pi}{2}$.

The error in $\theta_{s}$ due to the DFIG stator impedance voltage drop under load is negligible in practice. This is a significant advantage of indirect orientation scheme since the orientation is shielded from measurement noise and stator voltage harmonics [13].

The demodulation of the rotor current and modulation of the rotor demand voltage uses the slip angle derived from the classical frequency relationship of the induction machine.

$\theta_{r}=\theta_{s}-\theta_{m}$,

where $\theta_{m}$ is rotor angle position measured via speed encoder.

\section{Simulation results}

The proposed control strategy for DFIG based standalone power generation system is modeled and simulated in Matlab/Simulink software program. The detailed parameters of the simulated system are listed in Appendix. The performance and effectiveness of the proposed system is tested under various conditions such as disturbances in rotor speed, unbalanced load condition and non-linear load condition. The obtained results are discussed in the following subsections.

\subsection{Performance under rotor speed disturbances}

The objective of this test is to verify the system performance and the dynamic behavior of the stator voltage under generator variable speed condition. A linear-balanced three-phase load connected to the PCC is assumed to be constant and the generator speed is assumed to vary depending on the profile shown in Fig. 7. This profile has been selected to operate the generator below and above the synchronous speed. The results obtained under these test conditions are shown in Figs. 8 and 9.

The regulation capability of the proposed control strategy to maintain a constant output voltage during the whole speed profile can be observed in Fig. 8. It can be seen that the stator RMS voltage and the DC-link voltage are kept constant at their respective reference values, and the correct orientation of the stator flux vector is also achieved despite large variations in rotor speed. It can also be noted
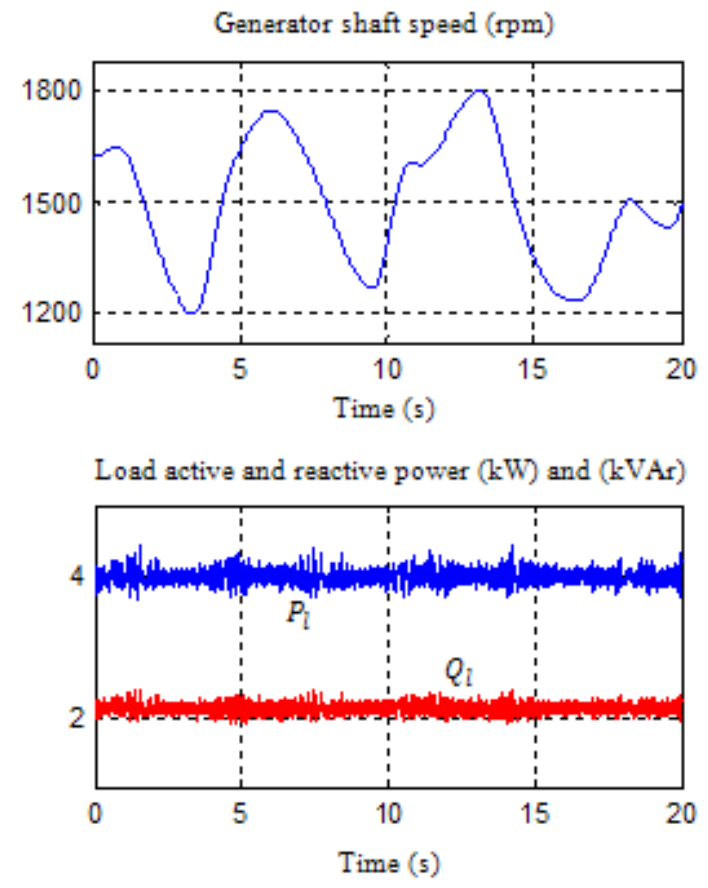

Fig. 7 System test conditions for rotor speed disturbances 

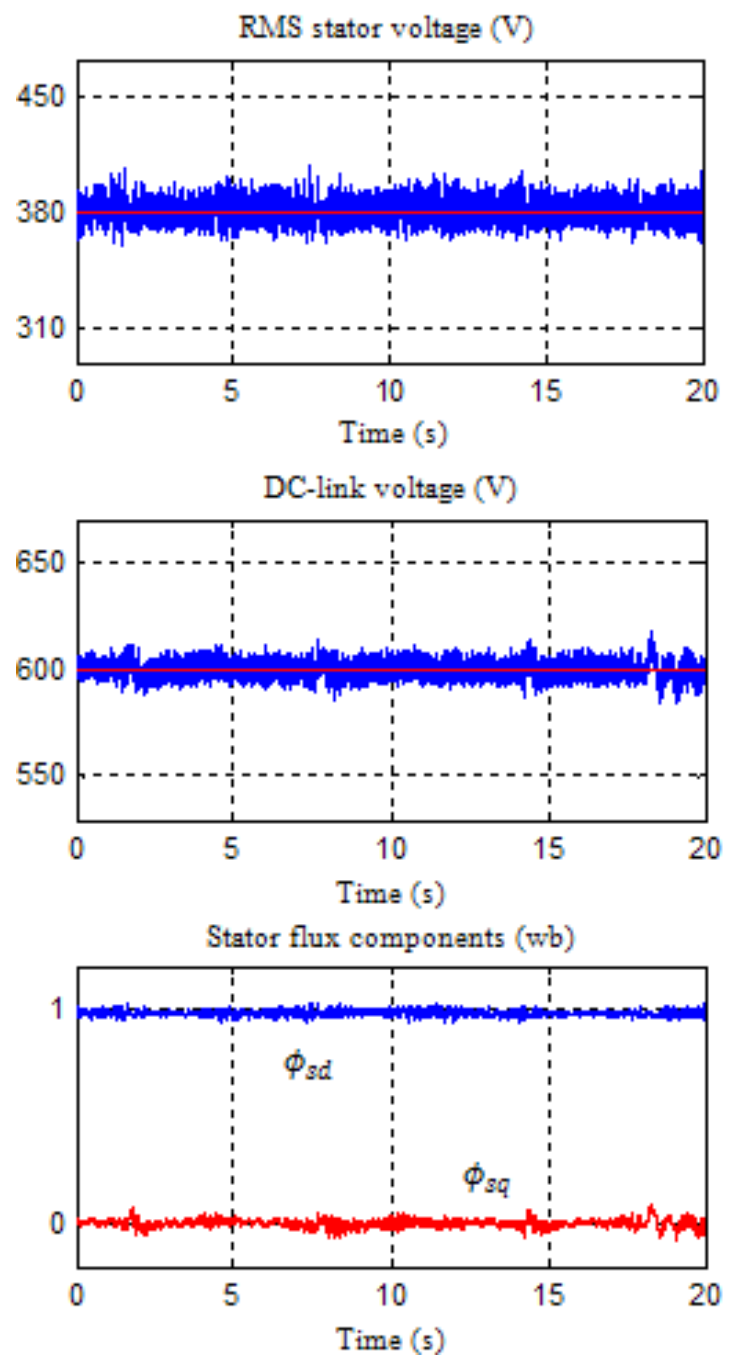

Fig. 8 Control performance under speed disturbances

that an instantaneous frequency of $50 \mathrm{~Hz}$ is maintained throughout the simulated duration, because it is directly fixed by the LSC control structure by applying a PCC voltage with a fundamental frequency equal to the stator voltage reference frequency. These results confirm that the variation of the generator speed has a negligible effect on the dynamics of the voltage of the generator, because of the slow process of variation of the speed of the rotor.

Fig. 9 illustrates the power flows in the system corresponding to the test conditions considered. It can be noted that at each instant the active power consumed by the load is equal to the sum of the active powers of the stator and the rotor. The distribution of the active powers is according to the rotor speed. Below the synchronous speed (hypo-synchronous mode), the rotor absorbs active power, which is generated by the stator, which causes an increase in the power contribution of the stator.

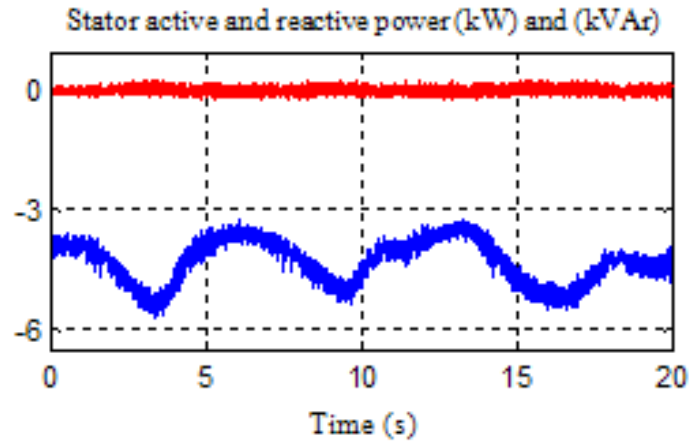

LSC active and reactive power (kW) and (kVAr)

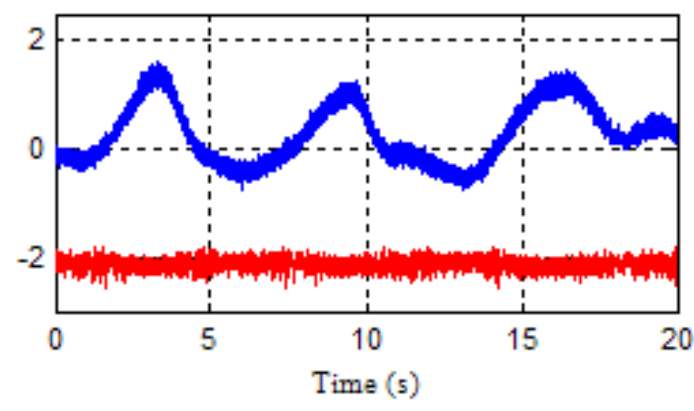

Fig. 9 Power flows during generator speed disturbances

Conversely, above the synchronous speed (hyper-synchronous mode), the flow of the rotor power is reversed, the stator and the rotor supply active power to the load, in this case, the stator is less charged. Finally, the reactive power of the stator is maintained zero throughout the simulation period, thereby confirming the operation of the stator side with a unitary power factor, and the required reactive power of the load is provided entirely by the LSC.

\subsection{Performance considering unbalanced load}

In this test, the generator is assumed to run at constant speed of $0.8 \mathrm{pu}$ under unbalanced load conditions using the proposed control strategy. Initially, the DFIG run under no-load condition and at $t=1 \mathrm{~s}$, an unbalanced $4.3 \mathrm{~kW}$ resistive load is connected to the three-phase stator terminals, in which the imbalance factor of the load current is $25 \%$. The obtained simulation results of the proposed control under the mentioned conditions are shown in Fig. 10.

The dynamic and steady state operation performances of the proposed control when load changes, are shown in Fig. 10. It can be seen that, based on the proposed control strategy, the amplitude and the frequency of the output voltage can be controlled constant in the condition of sudden load change. After the transient, it can be observed that the generator torque settles to a new operation point, for that the desired amount power will be delivered to 


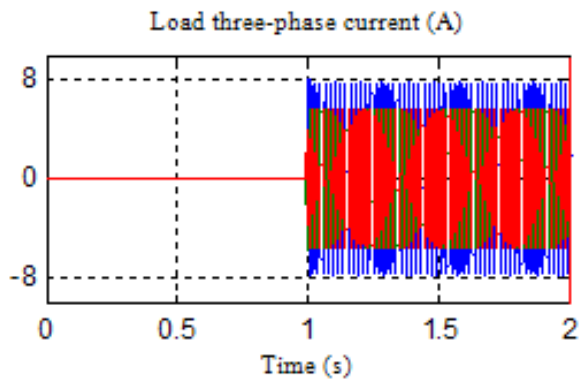

(a)

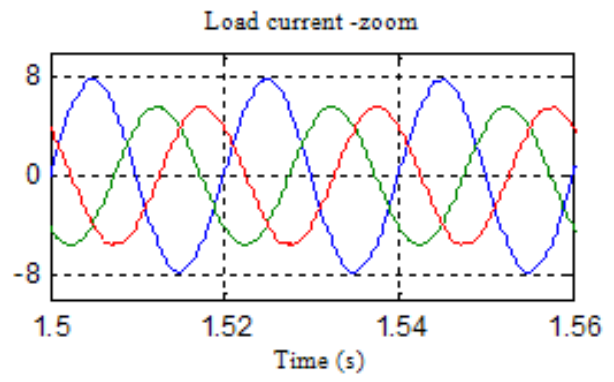

Stator three-phase current (A)

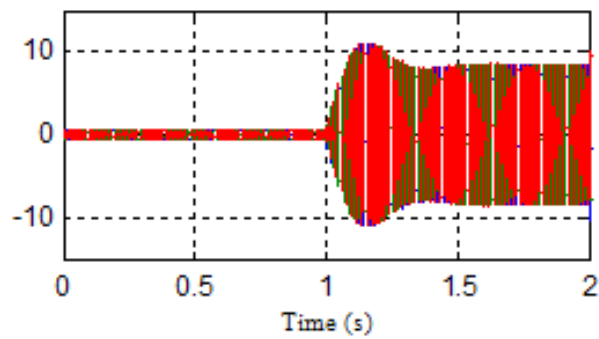

Stator current - zoom

(b)

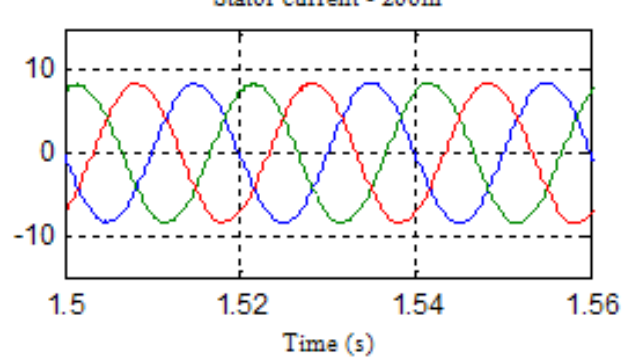

SC three-phase current (A)

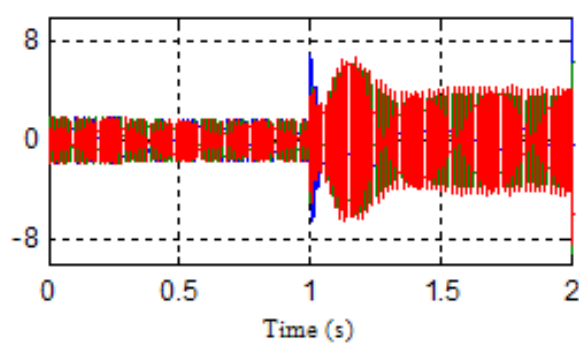

LSC current - zoom

(c)

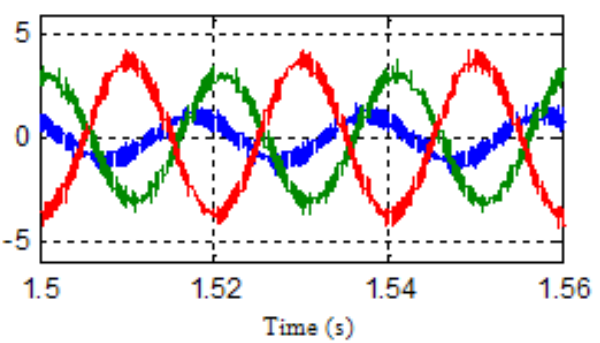

Stator three-phase voltage (V)

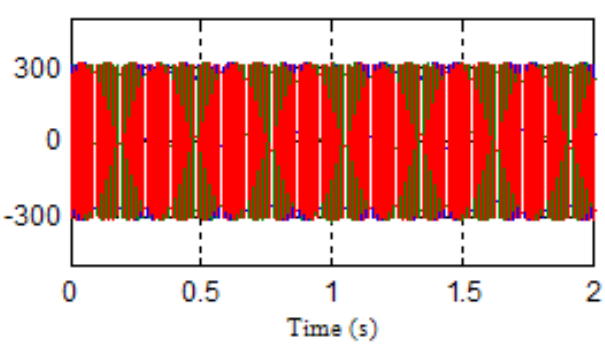

(d)
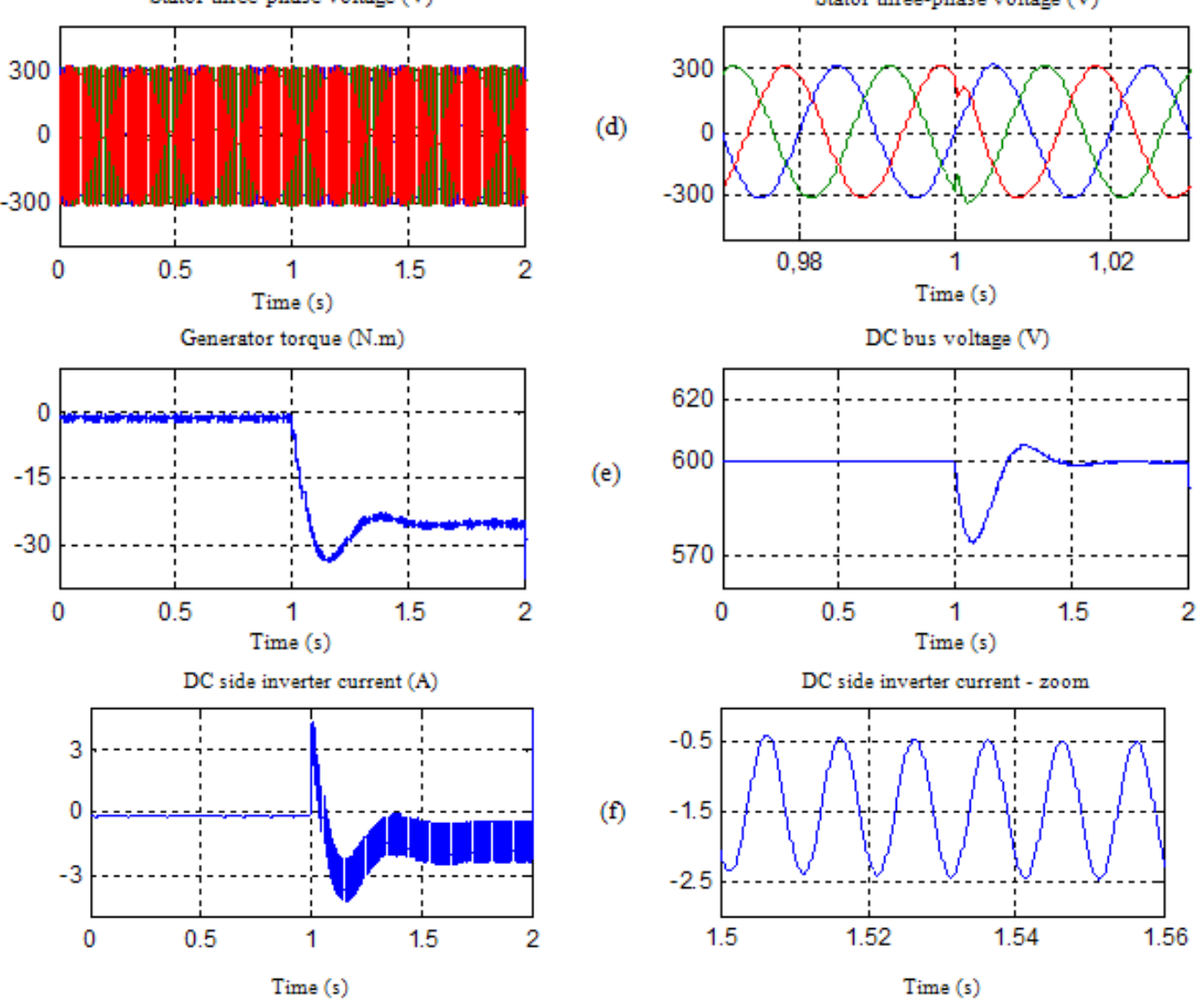

Fig. 10 Dynamic and steady state variables responses under unbalanced load 
the load, and the DC bus voltage is kept near its set point when steady state is reached, its excursion is within $6 \%$ during the load commutation.

According to analysis above, the PCC voltage and stator current would be unbalanced when unbalanced load takes place, because of negative sequence component. However, using the proposed compensation method applied for the LSC, the stator voltage at the PCC can be compensated well. As it can be observed in Fig. 10, the generated stator voltages seem fully balanced and exhibit excellent performance under unbalanced load. The unbalanced factor of the output voltage is only $1 \%$. It is clear from results that load current negative component is completely supplied by the LSC, which acts as a shunt active filter, as a result, the stator current become effectively compensated and waveforms of three phases are balanced.

Despite the pulsating of active power demanded by the load, the torque ripples seem fully eliminated from the generator, thanks to the elimination of negative sequence in the DFIG stator and rotor currents. As the pulsation component of demanded power is supplied from the LSC. Consequently, the DC side current of LSC contains the AC component of double-fundamental frequency. As shown in Fig. 10 (f), this double-fundamental frequency harmonic in the DC current can be anticipated when the decoupling between the two cascaded converters is fully realized by the DC bus capacitor.

\subsection{Performance considering nonlinear load}

In this section, test on the system control and operation during non-linear load conditions is carried out to demonstrate the validity of the proposed control. The system is assumed that is running under a fixed rotor speed with a balanced $6.1 \mathrm{~kW}$ nonlinear load, in which is implemented by connecting a three-phase full bridge diode rectifier with resistive loads at the PCC, which leads to total harmonic distortion of load current about $10.48 \%$. For this case, the LSC controller with a PRC tuned at the fundamental, the fifth and the seventh selected frequencies is designed for the stator voltage control. The RSC is controlled with the traditional vector control to maintain the power balance between the generator and load. The obtained simulation results are shown in Fig. 11.

As stated above, when nonlinear load takes place, both stator current and PCC voltage would be distorted and contain harmonics with $6 n \pm 1$ multiples of fundamental frequency. However, it can be seen that the proposed controller can provide accurate regulation of the output voltage. By using the resonant frequency $\omega_{s}, 5 \omega_{s}$ and $7 \omega_{s}$ in the PR regulator, fifth and seventh harmonic components at the PCC voltage are totally rejected, and the stator voltage quality is significantly improved. It can be observed from the harmonic spectrum of stator voltage that the THD is only $1.85 \%$.

The steady-state performance on the stator current with the proposed control is shown in Fig. 11. It can be seen that harmonic stator currents are fully compensated by using PRCs in the stationary reference frame. As mentioned previously, the LSC is considered as an active power filter that produces the filter current. As a result, a quasi sinusoidal stator current is obtained in the stator despite the presence of nonlinear load. The THD of the stator current is only $2.26 \%$. This means that the proposed method is able to eliminate the main components of the generator torque ripples, and this despite the presence of the sixth harmonic in the DC-side current shown in Fig. 11.

\section{Conclusion}

This paper has proposed a new control strategy to improve the quality of stator voltage and current at the PCC in a stand-alone DFIG system feeding unbalanced and nonlinear loads. In this loads conditions, the negative sequence and harmonic components of stator voltage and current are harmful for the generator system and also they would lead to degradation of power quality. Considering the adverse effect of voltage drops, torque pulsations and the need for an autonomous DFIG system, the elements controlling the power flows and attenuating harmonic and unbalanced components are also added as control targets for a DFIG stand-alone power generation system in this paper.

The proposed control strategy is developed in the LSC to regulate reactive power required by the load and rejects both harmonic and unbalanced components, so as to obtain good power quality and maximum active power generation capability; while the RSC regulates the DC bus voltage and adjusts the generator torque and therefore the balance of the stand-alone system. This control strategy based PRCs in the stationary reference frame is applied in LSC for compensating the unbalance and harmonic distortion in the stator voltage and current of the DFIG. This strategy is conceptually simple and his implementation does not complex, it does not require for rotating coordinate transformation and the sequence decomposition process. In addition, the RSC is controlled with the traditional vector control strategy to keep the DC bus voltage, which requires not current sensors to be installed at load or stator side, so that the number of current sensors employed 

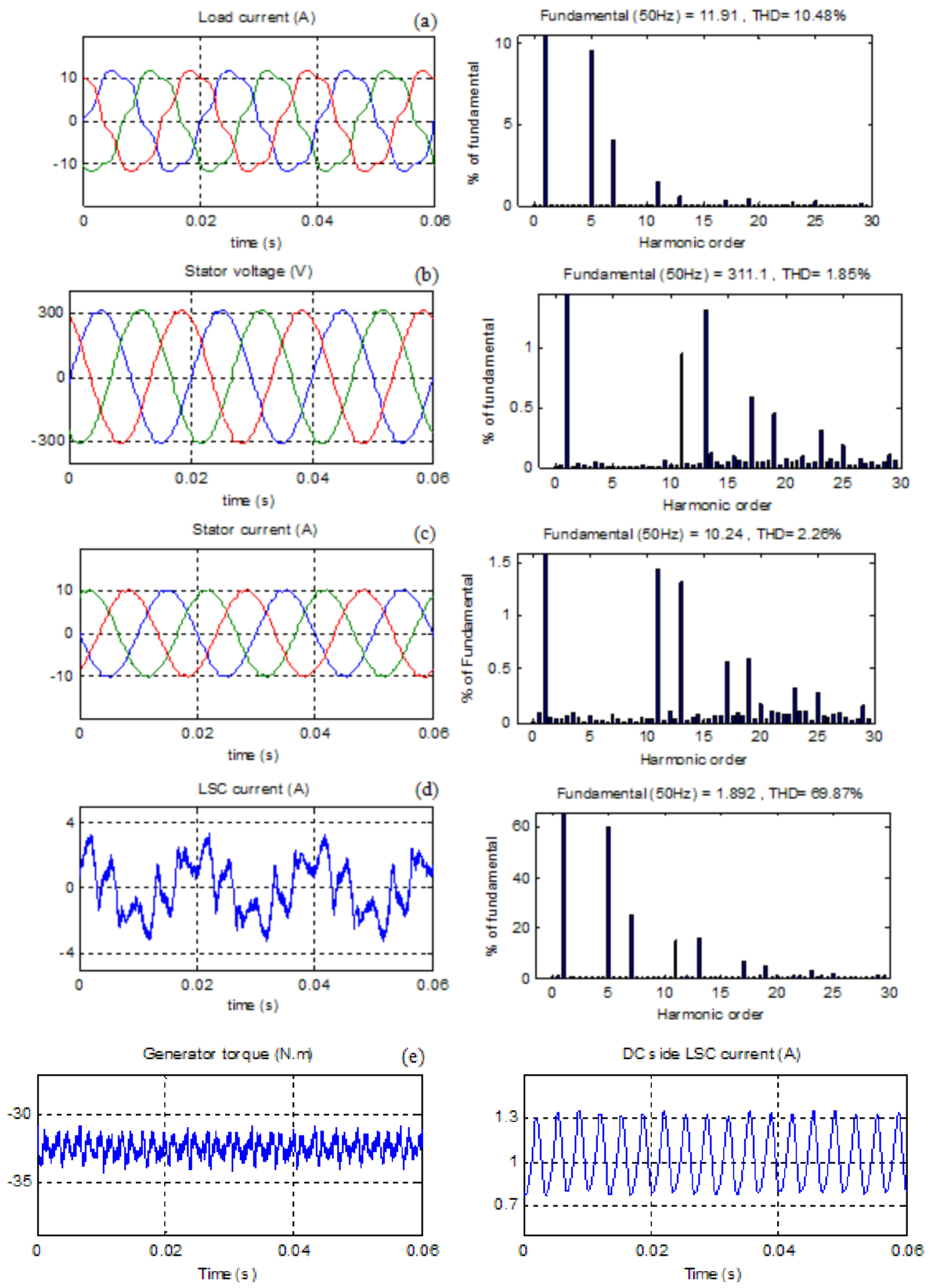

Fig. 11 Steady state control performance under nonlinear load 
in the system is reduced. The simulation results are shown a good performance of the proposed control strategy and evaluate the effectiveness of the compensating method.

\section{Symbols}

$V, I, \phi \quad$ Voltage, current and flux quantities, respectively

$C_{d c} \quad$ DC bus capacitor

$R_{s}, R_{r} \quad$ Machine resistance per phase

$L_{t}, C_{t} \quad$ Filter inductance and capacitor $p$

$\sigma$

$\omega_{s}, \omega_{m}, \omega_{r}$ Synchronous, rotational rotor, and slip speeds $\theta_{s}, \theta_{m}, \theta_{r}$ Synchronous, rotational rotor, and slip angles

$\Omega_{g}, T_{g} \quad$ Generator shaft speed and torque, respectively

$P, Q \quad$ Active and reactive power

$(d, q) \quad$ Synchronous rotating coordinates

$(x, y) \quad$ Stator fixed coordinates

$(a, b, c) \quad$ Stationary three-phase coordinates

\section{References}

[1] Aouzellag, D., Ghedamsi, K., Berkouk, E. M. "Network power flux control of a wind generator", Renewable Energy, 34(3), pp. 615-622, 2009.

https://doi.org/10.1016/j.renene.2008.05.049

[2] Chikha, S., Barra, K. "Predictive Control of Variable Speed Wind Energy Conversion System with Multi Objective Criterions", Periodica Polytechnica Electrical Engineering and Computer Science, 60(2), pp. 96-106, 2016.

https://doi.org/10.3311/PPee.8873

[3] Benbouhenni, H., Boudjema, Z., Belaidi, A. "Using Four-Level NSVM Technique to Improve DVC Control of a DFIG Based Wind Turbine Systems", Periodica Polytechnica Electrical Engineering and Computer Science, 63(3), pp. 144-150, 2019. https://doi.org/10.3311/PPee.13636

[4] Kara, Z., Barra, K. "Hybrid Controller for Variable Speed Wind Energy Conversion System with Slip Energy Recovery Using Matrix Converter Topology", Periodica Polytechnica Electrical Engineering and Computer Science, 59(4), pp. 160-174, 2015. https://doi.org/10.3311/PPee.8507

[5] Chen, J., Zhang, W., Chen, Y., Ma, Y. "Improved Vector Control of Brushless Doubly Fed Induction Generator under Unbalanced Grid Conditions for Offshore Wind Power Generation", IEEE Transaction on Energy Conversion, 31(1), pp. 293-302, 2016. https://doi.org/10.1109/TEC.2015.2479859

[6] Mishra, A., Tripathi, P. M., Chatterjee, K. "A review of harmonic elimination techniques in grid connected doubly fed induction generator based wind energy system", Renewable and Sustainable Energy Reviews, 89, pp. 1-15, 2018.

https://doi.org/10.1016/j.rser.2018.02.039

[7] Song, Y., Nian, H. "Sinusoidal Output Current Implementation of DFIG Using Repetitive Control Under a Generalized Harmonic Power Grid With Frequency Deviation", IEEE Transactions on Power Electronics, 30(12), pp. 6751-6762, 2015.

https://doi.org/10.1109/TPEL.2015.2390213

[8] Shukla, R. D., Tripathi, R. K. "Isolated Wind Power Supply System using Double-fed Induction Generator for remote areas", Energy Conversion and Management, 96, pp. 473-489, 2015. https://doi.org/10.1016/j.enconman.2015.02.084

[9] Abdoune, F., Aouzellag, D., Ghedamsi, K. "Terminal voltage build-up and control of a DFIG based stand-alone wind energy conversion system", Renewable Energy, 97, pp. 468-480, 2016. https://doi.org/10.1016/j.renene.2016.06.005

[10] Xu, W., Mohammed, O. M. E., Liu, Y., Islam, M. R. "Negative Sequence Voltage Compensating for Unbalanced Standalone Brushless Doubly-fed Induction Generator", IEEE Transactions on Power Electronics, 35(1), pp. 667-680, 2020.

https://doi.org/10.1109/TPEL.2019.2912820

[11] Jain, A. K., Ranganathan, V. T. "Wound Rotor Induction Generator With Sensorless Control and Integrated Active Filter for Feeding Nonlinear Loads in a Stand-Alone Grid", IEEE Transactions on Industrial Electronics, 55(1), pp. 218-228, 2008. https://doi.org/10.1109/TIE.2007.911196

[12] Ramos, C. J., Martins, A. P., Carvalho, A. S. "Rotor Current Controller with Voltage Harmonics Compensation for a DFIG Operating under Unbalanced and Distorted Stator Voltage", In: IECON 2007 - 33rd Annual Conference of the IEEE Industrial Electronics Society, Taipei, Taiwan, 2007, pp. 1287-1292. https://doi.org/10.1109/IECON.2007.4459938

[13] Pena, R., Cardenas, R., Escobar, E., Clare, J., Wheeler, P. "Control System for Unbalanced Operation of Stand-Alone Doubly Fed Induction Generators", IEEE Transactions on Energy Conversion, 22(2), pp. 544-545, 2007. https://doi.org/10.1109/TEC.2007.895393

[14] $\mathrm{Xu}, \mathrm{H} ., \mathrm{Hu}, \mathrm{J} ., \mathrm{He}, \mathrm{Y}$. "Integrated Modeling and Enhanced Control of DFIG Under Unbalanced and Distorted Grid Voltage Conditions", IEEE Transactions on Energy Conversion, 27(3), pp. 725-736, 2012. https://doi.org/10.1109/TEC.2012.2199495

[15] Phan, V. T., Lee, H. H. "Stationary frame control scheme for a stand-alone doubly fed induction generator system with effective harmonic voltages rejection", IET Electric Power Applications, 5(9), pp. 697-707, 2011. https://doi.org/10.1049/iet-epa.2010.0280

[16] Xu, W., Dong, D., Liu, Y., Yu, K., Gao, J. "Improved Sensorless Phase Control of Stand-Alone Brushless Doubly-Fed Machine Under Unbalanced Loads for Ship Shaft Power Generation", IEEE Transactions on Energy Conversion, 33(4), pp. 2229-2239, 2018. https://doi.org/10.1109/TEC.2018.2865594

[17] Phan, V. T., Lee, H. H. "Control Strategy for Harmonic Elimination in Stand-Alone DFIG Applications with Nonlinear Loads", IEEE Transactions on Power Electronics, 26(9), pp. 2662-2675, 2011. https://doi.org/10.1109/TPEL.2011.2123921 
[18] Cheng, M., Jiang, Y., Han, P., Wang, Q. "Unbalanced and Low-Order Harmonic Voltage Mitigation of Stand-Alone Dual-Stator Brushless Doubly Fed Induction Wind Generator", IEEE Transactions on Industrial Electronics, 65(11), pp. 9135-9146, 2018. https://doi.org/10.1109/TIE.2017.2779422

[19] Phan, V. T., Lee, H. H. "Performance Enhancement of Stand-Alone DFIG Systems with Control of Rotor and Load Side Converters Using Resonant Controllers", IEEE Transactions on Industry Applications, 48(1), pp. 199-210, 2012.

https://doi.org/10.1109/TIA.2011.2175883

[20] Hu, J., He, Y., Xu, L., Williams, B. W. "Improved Control of DFIG Systems During Network Unbalance Using PI-R Current Regulators", IEEE Transactions on Industrial Electronics, 56(2), pp. 439-451, 2009.

https://doi.org/10.1109/TIE.2008.2006952

[21] Hu, J., He, Y. "DFIG wind generation systems operating with limited converter rating considered under unbalanced network conditions - Analysis and control design", Renewable Energy, 36(2), pp. 839-847, 2011.

https://doi.org/10.1016/j.renene.2010.07.004

\section{Appendix}

Parameters of the simulated DFIG:

Rated voltage

Rated Power

$7.5 \mathrm{~kW}$

Nominal frequency

$50 \mathrm{~Hz}$

Number of poles

4 poles

Stator winding resistance
[22] Fan, L., Yuvarajan, S., Kavasseri, R. "Harmonic Analysis of a DFIG for a Wind Energy Conversion System", IEEE Transactions on Energy Conversion, 25(1), pp. 181-190, 2010. https://doi.org/10.1109/TEC.2009.2032594

[23] Nayeripour, M., Mahdi Mansouri, M. "An advanced analytical calculation and modeling of the electrical and mechanical harmonics behavior of doubly fed induction generator in wind turbine", Renewable Energy, 81, pp. 275-285, 2015. https://doi.org/10.1016/j.renene.2015.03.018

[24] Zeng, J., Degobert, P., Loriol, D., Hautier, J. P. "Robust design of the self-tuning resonant controller for AC current control systems", In: 2005 IEEE International Conference on Industrial Technology, Hong Kong, China, 2005, pp. 783-788. https://doi.org/10.1109/ICIT.2005.1600742

[25] Kazmierkowski, M. P., Malesani, L. "Current control techniques for three-phase voltage-source PWM converters: a survey", IEEE Transactions on Industrial Electronics, 45(5), pp. 691-703, 1998. https://doi.org/10.1109/41.720325

Rotor winding resistance

$0.620 \mathrm{Ohm}$

Magnetism inductance

$0.078 \mathrm{H}$

Stator inductance

$0.084 \mathrm{H}$

Rotor inductance

$0.081 \mathrm{H}$

DC-link capacitor

$500 \mu \mathrm{F}$ 